\title{
ZnO Nanoparticles to Nanowires and Nanobundles
}

\section{Mohammad Kamal Hossain ${ }^{1, \#, \$}$}

\author{
${ }^{1}$ The MacDiarmid Institute for Advanced Materials and Nanotechnology, School of Chemical and \\ Physical Science, Victoria University of Wellington, Wellington 600, New Zealand. \\ ${ }^{\#}$ Microelectronics, School of Engineering and Technology, Asian Institute of Technology, Klong \\ Luang, Pathumthani 12120, Thailand. \\ ${ }^{\$}$ Current address: Center of Research Excellence in Renewable Energy, King Fahd University of \\ Petroleum and Minerals, Dhahran 131261, Kingdom of Saudi Arabia. \\ kamalhossain@kfupm.edu.sa
}

Keywords: ZnO, nanoparticles, nanowires, nanobundles, sol-gel, anisotropic aggregation.

\begin{abstract}
Nanosized Zinc oxide $(\mathrm{ZnO})$ possesses unique electrical, optoelectronics and photochemical characteristics and thus it is a potential candidate for different applications in next generation of optoelectronic device. In this work, a novel sol-gel route for the synthesis of $\mathrm{ZnO}$ nanoparticles and its transformation into wires and bundles has been reported. The process is adopted from a simple and hand-on route that also shows the power of green chemistry in nanomaterials synthesis. $\mathrm{ZnO}$ nanoparticles $(\sim 30 \mathrm{~nm}$ in diameter) were synthesized from bottom-up approach followed by a further process to obtain nanometric wires and bundles under controlled conditions. The nanowires and bundles are speculated to initiate from anisotropic agglomeration of nanometric particles and agglomeration of these nanometric wires into bundles respectively. Control of these agglomeration processes is a key challenge for application of nanowires and bundles into useful devices.
\end{abstract}

\section{Introduction}

Zinc oxide $(\mathrm{ZnO})$ is well-known for its attractive and impressive characteristics such as direct wide band gap $(\sim 3.37 \mathrm{eV})$, good transparency $(\sim 90 \%)$, high electron mobility $\left(>100 \mathrm{~cm}^{2} / \mathrm{Vs}\right)$, improved photoconductivity, etc. [1-5]. It has attracted increasing attention due to its excellent optical and 
electrical properties, chemical stability towards air, ability to produce significant quantum confinement effect [5, 6-7]. Nanosized $\mathrm{ZnO}$ has immense importance in solar cells and electronic devices as well due to its thermal stability, irradiation resistance and flexibility to form different nanostructures $[7,8]$. To the extent, structures like nanowires, nanobelts and nanorings are of great interest in photonics, optoelectronics and nanotechnology [9-15]. Therefore, the controlled synthesis of various $\mathrm{ZnO}$ nanostructures such as nanocrystals, nanowires, nanobelts, nanobundles and other complex nano architectures are of great interest.

$\mathrm{ZnO}$ nanoparticles and its different structures have been studied extensively in different research laboratories such as Syn. X-ray lab., Berkeley lab., Kawasaki lab. etc. A wide range of techniques including chemical vapor deposition (CVD), metalorganic chemical vapor deposition (MOCVD), thermal pyrolysis, etc. are generally used to synthesize zinc oxide $(\mathrm{ZnO})$ nanoparticles and films [1618]. In most of the cases, it is reported that $\mathrm{ZnO}$ nanostructures are resulted from epitaxial growth rather than isotropic nucleation. Amongst the techniques available for synthesis of nanoparticles, the sol-gel route is very attractive, because it is relatively simple and allows the possibility to control the building blocks. The major problem in sol-gel synthesis route is the control of the hydrolysis rate, which is generally too fast, resulting in the formation of precipitates with a high degree agglomeration. The strategy frequently used to reduce the hydrolysis-condensation rate is the modification of reactivity of precursors by controlling additives.

In this report, selective growth of $\mathrm{ZnO}$ colloidal nanoparticles $\left(\mathrm{D}_{\mathrm{ZnO}} \approx 30 \mathrm{~nm}\right)$ at low temperature from sol-gel route has been presented followed by a control of forming $\mathrm{ZnO}$ nanowires and nanobundles. The nanowires and bundles obtained in this work are speculated to initiate from anisotropic agglomeration of nanometric particles and agglomeration of these nanometric wires into bundles respectively. $\mathrm{ZnO}$ nanoparticles were found to be pure hexagonal wurtzite structure with cell constants, $\mathrm{a}=3.2427 \AA$ and $\mathrm{c}=5.1948 \AA$. XRD indicates the planes of $\{100\}\{002\}$ and $\{101\}$ at $2 \theta=$ $31.9^{\circ}, 34.64^{\circ}$ and $36.42^{\circ}$ respectively. SEM micrographs show the indication of anisotropic agglomeration of $\mathrm{ZnO}$ nanoparticles to form nanowires and nanobundles thereof. 


\section{Methodology}

The optimization between surface charge and volume charge of the particles during neucleation process is very crucial to define size distribution of colloidal nanoparticles. Therefore, it is essensial to be very careful of ion concentration, such as $\mathrm{H}^{+} / \mathrm{OH}^{-}$of precursor solution. The process adopted herein can be broadly divided into three major steps:

(i) precursor preparation of zinc acetate solution,

(ii) colloidal nanoparticle formation by hydrolysis and

(iii) formation of nanometric wires and bundles from colloidal nanoparticles under controlled conditions.

The precursor was prepared from zinc acetate, $\mathrm{Zn}\left(\mathrm{CH}_{3} \mathrm{COO}\right)_{2} \cdot 2 \mathrm{H}_{2} \mathrm{O}$ (UNIVAR) and absolute ethanol (MERCK) with excess amount of deionized water water. $100 \mathrm{ml}, 0.1 \mathrm{M}$ solution was prepared from zinc acetate where ethanol and water were used as solvent. This ethanolic zinc acetate solution was refluxed for 1 hour at $80^{\circ} \mathrm{C}$ with magnetic stirrer. The flask was covered tightly to avoid exposure of moisture. The stock solution so-prepared was used as the precursor solution. The precursor, more likely Zinc hydroxy double salt $\left[\mathrm{Zn}_{5}(\mathrm{OH})_{8}\left(\mathrm{COOCH}_{3}\right)_{2}\right]$ was stored at $4{ }^{\circ} \mathrm{C}$ to avoid any precipitation.

The reaction flask was then placed in a reaction bath for speeding up the hydrolysis reaction. Different catalysts are reported to be used as catalyst to hydrolyze the precursor. It is also possible to carry out the hydrolysis without further addition of catalyst provided that there is excess water. As the precursor solution already contains excess water in this case, hydrolysis begins instantaneously. So we carried out the hydrolysis without addition of any catalysts as shown in Fig. 1. Fig. 1 shows a schematic synthesis diagram of three step process to form $\mathrm{ZnO}$ nanoparticels and its alignment into anisotropic aggregation to nanowires and nanobundles. Within 30 minutes to one hour colloidal particles are obtained which are fairly monodispersed as it is observed by SEM morphologies. 
Further to the synthesis of nanometric wires and bundles, the hydrolysis of the precursor solution was carried out in presence of glacial acetic acid as catalyst. At the same time, the $\mathrm{pH}$ of the precursor was maintained as 4.0. It is found that the catalist not only inhibits further formation of colloids, but also initiates anistropic aggregation of as-synthesized colloids rather than agglomeration.

Scanning electron microscopy (SEM, JSM-6301F), X-ray diffraction (XRD, JEOL JDX-3530), and UV-Vis spectrometer (UNICAM) were used to characterize the structure, morphology and the presence of oxide group in as-synthesized $\mathrm{ZnO}$ colloidal nanoparticles, apart from the usual equipments required for the synthesis and segregation of nanoparticles that include e.g. $\mathrm{pH}$ meter, ultrasonic baths, magnetic stirrer, ultracentrifuge, amongst others.

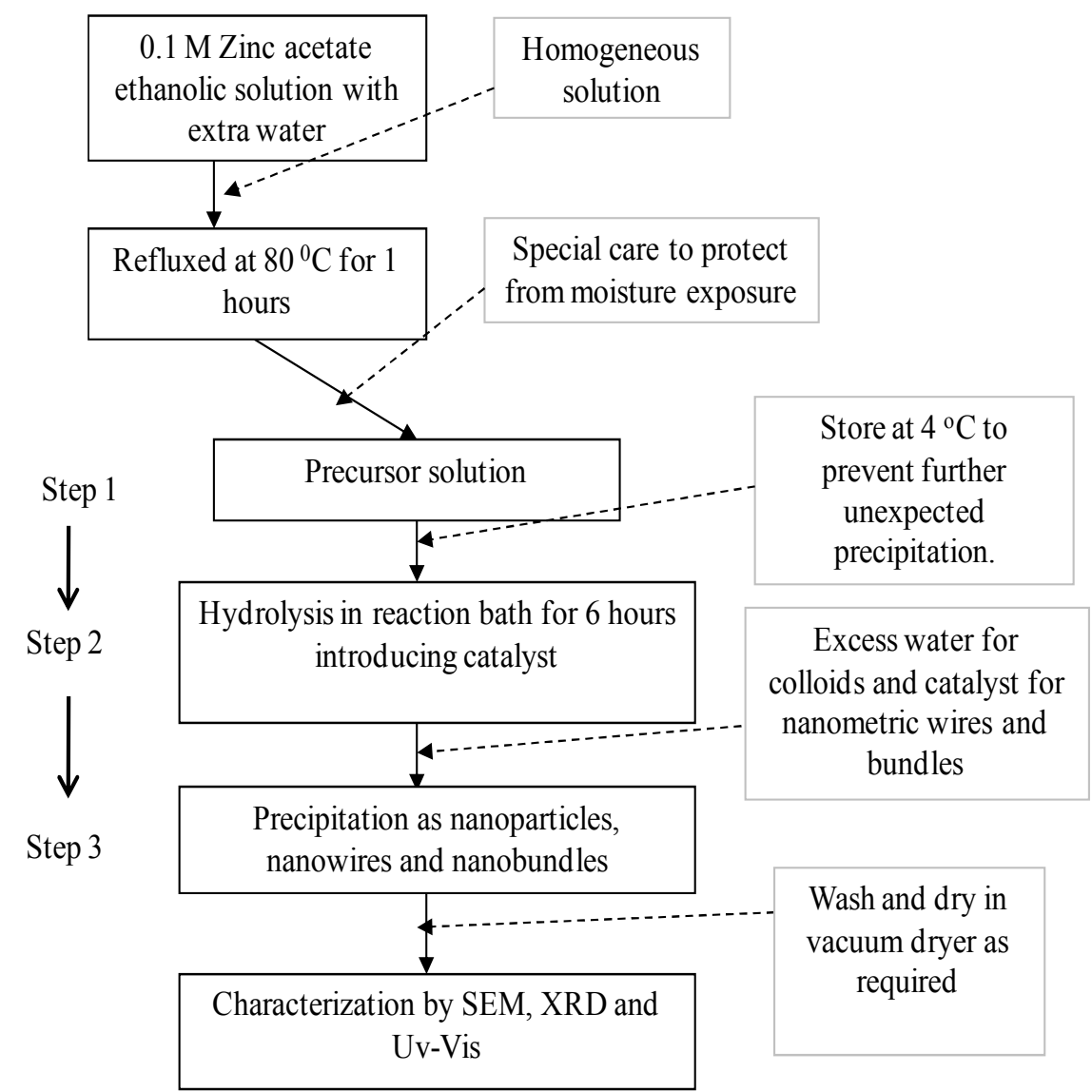

Fig. 1. Schematic flowchart for the synthesis of nanometric $\mathrm{ZnO}$ colloids and anisotropic aggregation as wires and bundles thereafter. Three steps are specifically pointed out to demonstrate the synthesis procedure. 


\section{Results and Discussion}

The growth of $\mathrm{ZnO}$ nanoparticles from ethanolic zinc acetate solution was confirmed initially from the UV-Vis spectra supported by SEM and XRD analysis. Fig. 2 shows SEM micrograph of fairly monodispersed $\mathrm{ZnO}$ nanoparticles. The upper inset is the UV-Vis spectra of precursor and that of colloidal suspension. A sharp change in absorption near $250 \mathrm{~nm}$ indicates begining of the nucleation of $\mathrm{ZnO}$ colloids.

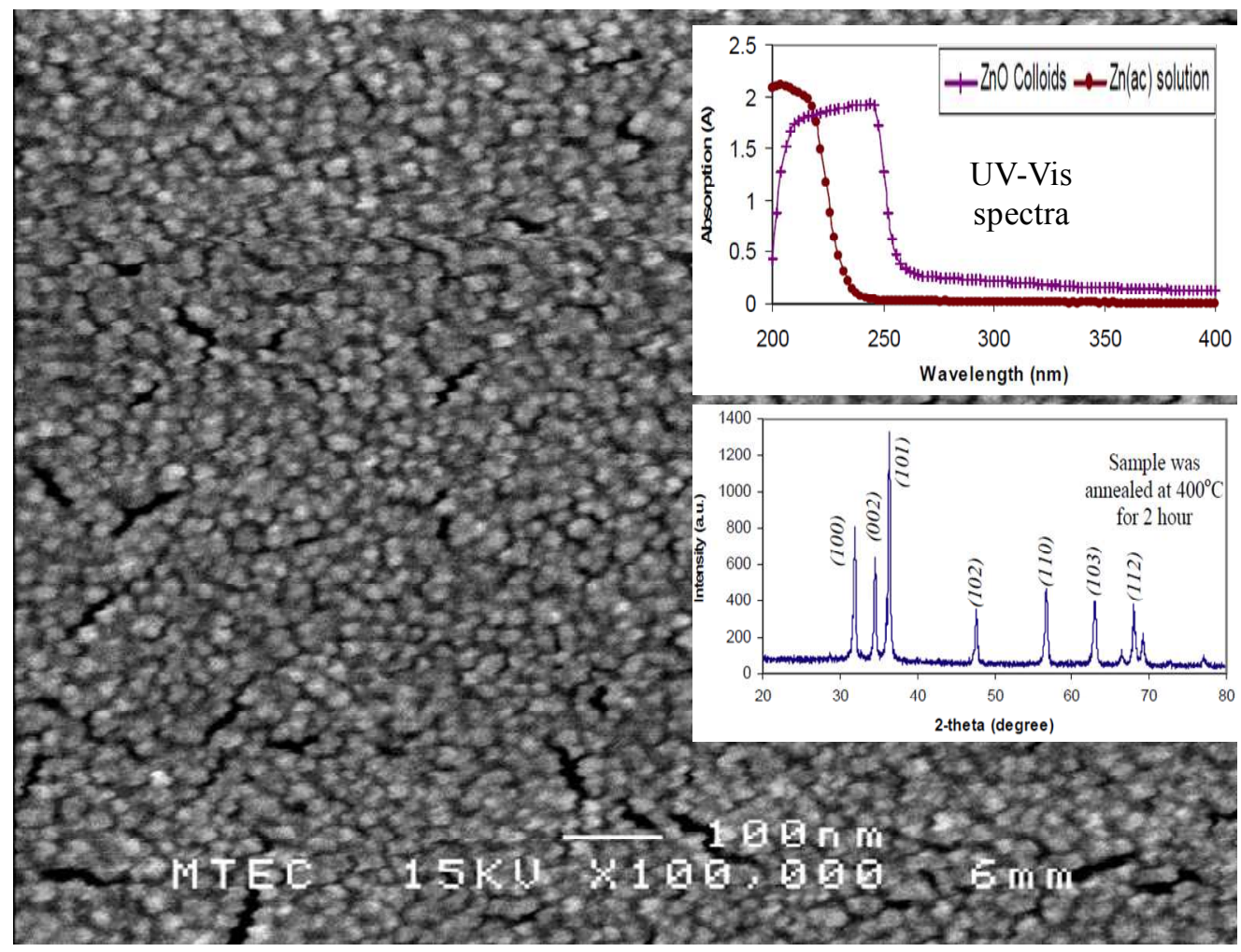

Fig. 2. SEM micrograph of $\mathrm{ZnO}$ nanoparticles showing fairly monodispersed colloids. Upper inset: UV-Vis spectra of precursor and that of colloidal suspension. Lower inset: XRD pattern indicating a pure hexagonal wurtzite crystal structure of $\mathrm{ZnO}$.

The lower inset represents XRD pattern indicating a pure hexagonal wurtzite crystal structure of $\mathrm{ZnO}$. The phase structure of the produced $\mathrm{ZnO}$ nanoparticles was identified by XRD. XRD measurement reveals that $\mathrm{ZnO}$ nanoparticles have hexagonal wurtzite crystal structure with the cell 
constants, $\mathrm{a}=3.2427 \AA$ and $\mathrm{c}=5.1948 \AA$. The diffraction peaks indicate planes $\{100\},\{002\}$ and $\{101\}$ at diffraction angle $2 \theta=31.9^{\circ}, 34.64^{\circ}$ and $36.42^{\circ}$ respectively as shown in upper inset of Fig.

2. No diffraction peaks from $\mathrm{Zn}$ or other impurities were found within the detection limit. The crystallite size has been calculated to be around $30 \mathrm{~nm}$ by using Debye-Scherrer formula.

As mentioned before, the nucleation of $\mathrm{ZnO}$ nanoparticles begins during hydrolysis and the hydrolysis process is very sensitive to $\mathrm{pH}$, catalyst, temperature, etc. Several SEM morphologies confirms that if the catalyst is added within one hour maintaining the $\mathrm{pH}$ at 4.0 , the colloidal nanoparticles begin to align and undergoes an anisotropic agglomeration to form nanowires. Addition of a catalyst was found to inhibit the isotropic agglomeration of particles, so particles do not agglomerate further. Instead, an anisotropic agglomeration occurs which lead to the formation of nanometric wires as shown in Fig. 3. Further refluxing resulted in nanobundles initiated by the agglomeration of zinc oxide $(\mathrm{ZnO})$ nanometric wires. Fig. 3 (a) shows a SEM micrograph of $\mathrm{ZnO}$ nanowire hanging away off a nanobundle. A single nanowire of more than a micron length is shown in Fig. 3 (b) and high resolution SEM image of the same nanowire is shown in inset. It is noteworthy that the constituent nanopartices are apperant, though not clear enough because of ionization effect surrounding the wire.
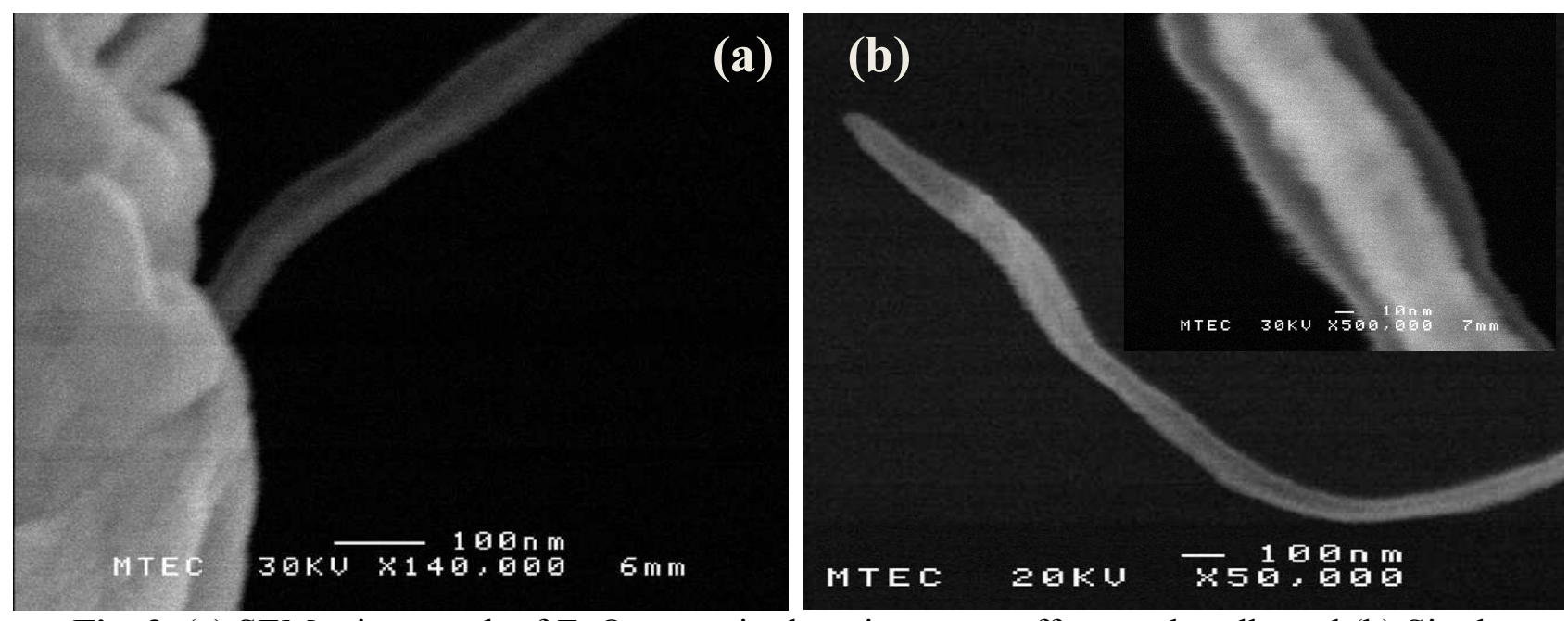

Fig. 3. (a) SEM micrograph of $\mathrm{ZnO}$ nanowire hanging away off a nanobundle and (b) Single nanowire of micron length. Inset: High resolution SEM image of the same nanowire showing anisotropic aggregation of colloids as constituent elements of the same. 


\section{Conclusion}

It is observed that the formation process of $\mathrm{ZnO}$ nanometric wires and bundles from colloidal nanoparticles in this work is strongly dependent on temperature, hydrolysis time, $\mathrm{pH}$, ion concentration of precursor and nature of the catalyst used in hydrolysis. The nucleation to colloidal nanoparticles starts without any inclusion of catalyst, whereas the catalyst inhibits the particles agglomerationa process and induces anisotropic alingment of the colloidal nanoparticles yielding nanowires and nanobundles. It is true that a lot of investigation is still required for better understanding of the mechanism of nanowires and bundles formation, but this process shows much promises for controlled growth of $\mathrm{ZnO}$ nanowires and bundles from colloidal nanoparticles.

\section{Acknowledgement}

The author likes to acknowledge Dr. J. Dutta, Dr. C. Thanachayanont. Dr. S.K. Rakshit, Dr. S.C. Ghosh and D. Jadsadapattarakul (Asian Institute of Technology, Thailand) for their support in experiment and characterization. Dr. H.A. AlHamayel and Dr. N. Tabet (CoRERE, King Fahd University of Petroleum and Minerals, Saudi Arabia) are also acknowledged for their support. National metal and materials technology centre (MTEC), Thailand is acknowledged for support in characterization. 


\section{References}

[1] R. Kaur, A.V. Singh, K. Sehrawat, N.C. Mehra, R.M. Mehra, Sol-gel derived yttrium doped ZnO nanostructures, J. Non-Cryst. Solids 352 (2006) 2565-2568.

[2] J. Kubota, K. Haga, Y. Kashiwaba, H. Watanabe, B.P. Zhang, Y. Segawa, Characteristics of ZnO whiskers prepared from organic-zinc, Appl. Surf. Sci. 216 (2003) 431-435.

[3] S. Singh, P. Thiyagarajan, K.M. Kant, D. Anita, S. Thirupathiah, N. Rama, B. Tiwari, M. Kottaisamy, M.S.R. Rao, Structure, microstructure and physical properties of $\mathrm{ZnO}$ based materials in various forms: bulk, thin film and nano, J. Phys. D: Appl. Phys. 40 (2007) 6312-6327.

[4] P.K. Giri, S. Bhattacharyya, B. Chetia, S. Kumari, D.K. Singh, P.K. Iyer, High-yield chemical synthesis of hexagonal $\mathrm{ZnO}$ nanoparticles and nanorods with excellent optical properties, J. Nanosci. Nanotech. 11 (2011) 1-6.

[5] J. Carrey, H. Carrere, M.L. Kahn, B. Chaudret, X. Marie, M. Respaud, Photoconductivity of self-assembled $\mathrm{ZnO}$ nanoparticles synthesized by organometallic chemistry, Semicond. Sci. Technol. 23 (2008) 025003-025008.

[6] R. Hong, T. Pan, J. Qian, H. Li, Synthesis and surface modification of ZnO nanoparticles, Chem. Eng. J. 119 (2006) 71-81.

[7] C. Wu, X. Qiao, J. Chen, H. Wang, F. Tan, S. Li, A novel chemical route to prepare ZnO nanoparticles, Mater. Lett. 60 (2006) 1828-1832. 
[8] D.C. Reynolds, D.C. Look, B. Jogai, Fine structure on the green band in ZnO, J. Appl. Phys. 89 (2001) 6189-6191.

[9] K. Westermark, H. Rensmo, A.C. Lees, J.G. Vos, H. Siegbahn, Electron spectroscopic studies of Bis-(2,2`-bipyridine)-(4,4'-dicarboxy-2,2'-bipyridine)-ruthenium(II) and Bis(2,2`-bipyridine)-(4,4`-dicarboxy-2,2`-bipyridine)-osmium(II) adsorbed on nanostructured TiO2 and ZnO surfaces, Phys. Chem. B 106 (2002) 10108-10113.

[10] Y. Zhang, K. Yu, D. Jiang, Z. Zhu, H. Geng, L. Luo, Zinc oxide nanorod and nanowire for humidity sensor, Appl. Surf. Sci. 242 (2005) 212-217.

[11] Q.H. Li, T. Gao, Y.G. Wang, T.H. Wang, Adsorption and desorption of oxygen probed from ZnO nanowire films by photocurrent measurements, Appl. Phys. Lett. 86 (2005) 123117123119.

[12] Z. Fan, D. Wang, P.C. Chang, W.Y. Tseng, J.G. Lu, ZnO nanowire field-effect transistor and oxygen sensing property, Appl. Phys. Lett. 85 (2004) 5923-5925.

[13] Z. Fan, J.G. Lu, Electrical properties of $\mathrm{ZnO}$ nanowire field effect transistors characterized with scanning probes, Appl. Phys. Lett. 86 (2005) 032111-032113.

[14] M.H. Huang, S. Mao, H. Feick, H.Q. Yan, Y.Y. Wu, H. Kind, E. Weber, R. Russo, P.D. Yang, Room-temperature ultraviolet nanowire nanolasers, Science 292 (2001), 1897-1899.

[15] M. Salavati-Niasari, N. Mir, F. Davar, ZnO nanotriangles: Synthesis, characterization and optical properties, J. Alloys and Comp. 476 (2009) 908-912. 
[16] M. Adachi, S. Tsukui, K. Okuyama, Nanoparticle Formation mechanism in CVD reactor with ionization of source vapor, J. Nanoparticles Res. 5 (2003) 31-37.

[17] S. Muthukumar, H. Sheng, J. Zhong, Z. Zhang, N.W. Emanetoglu, Y Lu, Selective MOCVD growth of ZnO nanotips, IEEE Trans. on Nanotech. 2 (2003) 50-54.

[18] A. Sanchez-Juarez, A. Tiburcio-Silver, A. Ortiz, E.P. Zironi, J. Rickards, Electrical and optical properties of fluorine-doped $\mathrm{ZnO}$ thin films prepared by spray pyrolysis, Thin Solid Films 333 (1998) 196-202. 